\title{
Seroprevalence of contagious caprine pleuropneumonia in Borana and Guji lowlands, Southern Ethiopia
}

\author{
Tesfaye Bekele ${ }^{1}$, Yilikal Asfaw ${ }^{2}$,Berhe Gebre-Egziabeher ${ }^{3}$, Getachew Abebe ${ }^{4}$ \\ ${ }^{1}$ Oromia Pastoralist Areas Development Commission, Addis Ababa, Ethiopia, E-mail address: \\ tesfa_me@yahoo.com, \\ ${ }^{2}$ Addis Ababa University, Faculty of Veterinary Medicine, Debre Zeit, Ethiopia, PO BOX 34 \\ ${ }^{3}$ Ministry of Agriculture of the Federal Democratic Republic of Ethiopia, Addis Ababa \\ ${ }^{4}$ Food and Agricultural Organization of United Nations, Addis Ababa, Ethiopia
}

\begin{abstract}
A multistage cross sectional serological study and questionnaire survey were conducted on contagious caprine pleuropneumonia in selected districts of Borana and Guji lowlands, Southern Ethiopia, to determine the prevalence of the disease and identify risk factors associated with the occurrence of the disease. A total of 900 sera samples were collected and tested using Complement Fixation Test (CFT). Questionnaire surveys were conducted with 69 randomly selected households. Out of the 900 goat sera samples tested, 119 (13.2\%) were seropositive for CCPP, giving an overall seroprevalence of $13.2 \%(95 \% \mathrm{CI}=11.0 \%-15.4 \%)$ in the study areas. A seroprevalence of $18.3 \%$ (95\% CI=14.3\%-22.7\%), $11.7 \%$ (95\% $\mathrm{CI}=8 \%-15.2 \%)$ and $9.7 \%(95 \% \mathrm{CI}=6.3 \%-12.6 \%)$ were recorded in Liban, Teltale and Moyale districts respectively. The seroprevalence recorded in Liban district was significantly different from that of Moyale district $(\mathrm{p}<0.05)$. Multivariate logistic regression analysis on the assumed risk factors showed that age, flock size and distance from veterinary service centre were the major risk factors associated with the occurrence of the disease with Odds ratios of 2.18 (95\% CI=1.64-2.91), 1.59 (95\% CI=1.11-2.29) and $1.43(95 \% \mathrm{CI}=1.03-1.98)$ respectively. The questionnaire survey has revealed that contact at watering points, restocking, lack of veterinary service, and large flock size were the major factors for the spread and occurrence of the disease in the area. In conclusion, the serological findings and questionnaire survey indicated that CCPP is the top major goat health problem in the area which warrants appropriate measures to be in place towards the prevention and control of the disease in the study areas.
\end{abstract}

Keywords: Borana, CCPP, Ethiopia, Guji, Seroprevalence. 


\section{Introduction}

Contagious caprine pleuropneumonia (CCPP) is a highly contagious, infectious fibrinous pleuropneumonia of goats caused by Mycoplasma capricolum subsp. capripneumoniae (Mccp),characterized by fever, respiratory distress with coughing, nasal discharge, high morbidity and mortality rates (Radostitis et al., 2006). Its economic importance is due to direct loss which result from its high mortality, reduced milk yield, cost of treatment and vaccination of the disease and indirect loss due to the imposition of trade restrictions. It has been reported from more than 35 countries most of which are in Africa. However, the exact distribution of the disease is not yet known mainly due to the lack of sensitivity and specificity of the diagnostic tests and difficulty of identification of the organism causing the disease (Nicholas, 2002). Since then, it has been recorded in different parts of the country where extensive type goat production system is practiced including Borana, Ogaden, Arsi, Gojam, East Shoa, South Omo and North Omo zones (Thiaucourt et al, 1992). In Borana and Guji lowlands of Southern Ethiopia, there have been many outbreaks of CCPP during the past years. Disease outbreak investigations and isolation of the causative agents has been conducted several times during the past. However, information on seroprevalence and associated risk factors were scanty. Therefore, the objectives of this study were to determine the seroprevalence of CCPP in the area and to identify the risk factors responsible for the occurrence of the disease.

\section{Materials and Methods}

\section{Description of the study area}

The study area comprises of Borana and Guji lowland of Southern Ethiopia, covering an area of $95000 \mathrm{~km}^{2}$. The Borana plateau gently slopes from the high mountain massif in the North 1650 metres above sea level to the South bordering Kenya at 1000 metres above sea level with slight variation due to central mountain ranges and scattered volcanic cones and craters (Coppock, 1994). The area borders Kenya to the South, Somali region to the East, the high land parts of Borana and Guji to the North, and Southern Nations and Nationalities People's Regional State to the West. The study was conducted in three selected districts, namely Teltale, Moyale and Liban. 


\section{Study design and sampling strategy}

The study designed was a cross sectional survey of all age and sex groups of goats in the study districts. A multistage sampling technique was employed. Accordingly, blood samples were collected from a total of 900 goats. The samples were tested using Complement Fixation Test (CFT) according to standard test procedure (OIE, 2004) at the National Veterinary Institute (NVI), Ethiopia. Questionnaire was administered to 69 randomly selected households.

\section{Categorization of study variables}

The ages of individual goats were determined according to Mike (1996) and were categorized into three groups as age group $\leq 2$ years (young), age group $>2$ years and $\leq 5$ years (adult) and age group $>5$ years (old). Goat flock sizes were categorized as $\leq 50$ goats (small flock size), $\geq 51$ and $\leq 160$ goats (medium flock size) and $\geq 161$ goats (large flock size). The distance from veterinary service centre was categorized into three groups based on previous studies by MoARD (2003) in the area, questionnaire survey and interview with the district veterinary officials. Accordingly, areas $\leq 10 \mathrm{kms}$ way from veterinary service centre (accessible), areas $>10 \mathrm{kms}$ to $\leq 30 \mathrm{kms}$ (moderately accessible) and areas $>30 \mathrm{kms}$ (inaccessible).

\section{Data analysis}

The associations of the individual risk factors with out come of interest were analyzed using Pearson's Chi-square, in SPSS (2006) version 15.00. The strength of association between the risk factors and the occurrence of the disease was assessed using Odds Ratio (OR) at the 95\% confidence level. The significant risk factors were further subjected to multivariable stepwise logistic regression analysis to determine the major risk factors. The major risk factors were used for the model construction to predict the occurrence of the disease in the study area

\section{Result}

An over all seroprevalence of $13.2 \%$ (95\% CI= 11.0\%-15.4\%) was observed in the study areas (Table 1). Seroprevalence of 11.7\% (95\% CI= 8.1\%-15.3\%), 9.7\% (95 $\mathrm{CI}=6.4 \%-13.0 \%)$ and $18.3 \%(95 \% \mathrm{CI}=14.0 \%-22.6 \%)$ were observed in Teltale, Moyale and Liban districts respectively. The differences in seroprevalences among the districts were statistically significant $(\mathrm{p}<0.05)$. No statistically significant difference observed between Moyale and Teltale districts ( $p>0.05)$. 
Pair wise comparisons showed significant difference existed between Liban and Moyale Districts $(\mathrm{p}<0.05)$.

Table 1. Overall seroprevalence of contagious caprine pleuropneumonia

\begin{tabular}{lccccc}
\hline District & $\begin{array}{c}\text { Sample } \\
\text { tested }\end{array}$ & $\begin{array}{c}\text { Sample } \\
\text { positive }\end{array}$ & $\begin{array}{c}\text { Prevalence (\%) } \\
\mathbf{( 9 5 \% C I )}\end{array}$ & P-value & $\begin{array}{c}\text { OR } \\
(\mathbf{9 5 \%} \text { CI) }\end{array}$ \\
\hline Moyale & 300 & 29 & $9.7(6.4-13.0)$ & & 1 \\
Teltale & 300 & 35 & $11.7(8.1-15.3)$ & 0.43 & $0.81(0.48-1.36)$ \\
Liban & 300 & 55 & $18.3(14.0-22.6)$ & 0.002 & $2.09(1.29-3.39)$ \\
Overall & 900 & 119 & $13.2(11.0-15.4)$ & & \\
\hline
\end{tabular}

The summary of seroprevalence based on the assumed risk factors is depicted in Table 2. Accordingly, seroprevalence recorded in female was $14 \%(95 \%$ $\mathrm{CI}=11.3 \%-16.7 \%)$, while it was $11.6 \%(95 \% \mathrm{CI}=8.0 \%-15.4 \%)$ in male. The seroprevalence between the two sexes was not significantly different $(\mathrm{p}>0.05)$. Similarly, seroprevalence of $9.2 \%$ (95\% CI=6.3\%-12.0\%), $11.0 \%$ (95\% CI=8.0\%$14.0 \%)$ and $34.8 \%(95 \% \mathrm{CI}=26 \%-42.8 \%)$ were recorded in young, adult age and old age categories respectively. The differences in seroprevalence among the age categories were significant $(p<0.05)$. Old age category has showed significantly different seroprevalence as compared to the young age category $(\mathrm{p}<0.05)$. The likelihood of the disease in old age category was 5.27 (95\%CI=3.13-8.87) times more likely to occur as compared to the young age category. Moreover, seroprevalence of $9.6 \%$ (95\% CI=5.5\%-13.5\%), 12\% (95\% CI=9.6\%-14.5\%) and $25 \%$ (95\% CI=17.3\%-32.7\%) was observed in small flock size, medium flock size and large flock size categories respectively. The difference in seroprevalence among the three flock size categories was significant $(p<0.05)$. Large flock size category has shown significantly different $(p<0.05)$ seroprevalence as compared to the small flock size category. The likelihood of the disease in large flock size category was $3.14(95 \% \mathrm{CI}=1.66-5.96)$ times more likely to occur as compared to the small flock size. Furthermore, seroprevalence of $10.6 \%$ (95\% CI $=6.6 \%$ $14.6 \%), 11.4 \%(95 \% \mathrm{CI}=8.7 \%-14.6 \%)$ and $21.9 \%(95 \% \mathrm{CI}=15.9 \%-27.9 \%)$ was observed in accessible, moderately accessible and inaccessible to veterinary service centre respectively. The difference in seroprevalence among the three distance categories was significant $(p<0.05)$. Inaccessible area has shown significant difference $(p<0.05)$ seroprevalence as compared to the accessible area. The likelihood of the disease in an inaccessible area was 2.36 (95\% $\mathrm{CI}=1.31-4.25)$ times more likely to occur as compared to the accessible area. 
Table 2. Seroprevalence of CCPP by assumed risk factors

\begin{tabular}{|c|c|c|c|c|c|c|}
\hline $\begin{array}{l}\text { Assumed } \\
\text { risk factors }\end{array}$ & Category & $\begin{array}{c}\text { Sample } \\
\text { tested }\end{array}$ & $\begin{array}{l}\text { Sample } \\
\text { positive }\end{array}$ & $\begin{array}{c}\text { Prevalence } \\
(\%) \\
(95 \% \mathrm{CI}) \\
\end{array}$ & P-value & $\begin{array}{c}\text { OR } \\
(95 \% \mathrm{CI})\end{array}$ \\
\hline \multirow[t]{2}{*}{ Sex } & Female & 599 & 84 & $14.0(11.3-16.7)$ & & $0.807(0.529-1.229)$ \\
\hline & Male & 301 & 35 & $11.6(8.0-15.2)$ & & \\
\hline \multirow[t]{3}{*}{ Age } & Young & 380 & 35 & $9.2(6.3-12.0)$ & & 1 \\
\hline & Adult & 408 & 45 & $11.0(8.0-14.0)$ & 0.398 & $1.22(0.767-1.94)$ \\
\hline & Old & 112 & 39 & $34.8(26.0-42.8)$ & 0.000 & $5.27(3.13-8.87)$ \\
\hline \multirow[t]{3}{*}{ Flock size } & Small & 188 & 18 & $9.6(5.5-13.5)$ & & 1 \\
\hline & Medium & 592 & 71 & $12.0(9.6-14.5)$ & 0.363 & $1.29(0.746-2.22)$ \\
\hline & Large & 120 & 30 & $25.0(17.3-32.7)$ & 0.000 & $3.14(1.66-5.95)$ \\
\hline \multirow{4}{*}{$\begin{array}{l}\text { Accessibility } \\
\text { to } \\
\text { veterinary } \\
\text { service }\end{array}$} & Accessible & 188 & 20 & $10.6(6.6-14.6)$ & & 1 \\
\hline & $\begin{array}{l}\text { Moderately } \\
\text { accessible }\end{array}$ & 543 & 62 & $11.4(8.7-14.6)$ & 0.77 & $1.08(0.63-1.85)$ \\
\hline & Inaccessible & 169 & 37 & $21.9(15.9-27.9)$ & 0.004 & $2.36(1.31-4.25)$ \\
\hline & Overall & 900 & 119 & $13.2(11.0-15.4)$ & & \\
\hline
\end{tabular}

Multivariable logistic regression analysis of the assumed risk factors showed that age group, flock size and distance from veterinary service centre were found to be statistically significant major risk factors $(p<0.05)$ responsible for the occurrence of the disease in the study area (Table 3). Hence the regression model for the occurrence of CCPP in the study area based on the major risk factors identified would be $\log (p)=-4.973+0.781$ Age +0.466 Flock size + 0.355 Distance from veterinary service centre.

Table 3. Multivariate logistic regression analysis for the major risk factors

\begin{tabular}{|c|c|c|c|}
\hline Variables & Coefficient of regression (B) & P value & OR (95\% CI) \\
\hline Age & 0.781 & 0.000 & $2.18(1.64-2.91)$ \\
\hline Flock size & 0.466 & 0.011 & $1.59(1.11-2.29)$ \\
\hline Distance & 0.355 & 0.034 & $1.43(1.03-1.98)$ \\
\hline Constant & -4.973 & 0.000 & 0.007 \\
\hline
\end{tabular}

The questionnaire survey indicated that contact at watering point, restocking, lack of veterinary service, contact at marketing points, large flock size and contact at grazing were identified as the major factors responsible for the occurrence and spread of the disease in the area. Accordingly, contact of goats 
at watering (24\%), restocking (19\%) and lack of veterinary service in the area (18\%) were identified to be the first three most important factors for the spread and occurrence of CCPP disease in the area in descending order.

\section{Discussion}

The overall seroprevalence of CCPP in the study areas was $13.2 \%(\mathrm{CI}=11.0 \%$ $15.5 \%)$. The finding in the present study is about similar to the work of Solomon and Kassahun (2009) who reported seroprevalence of 18.61\% in South Omo and Arbaminch areas. However, it was higher than that of Beyene Nigatu (2003) and lower than that of Gezahegn Eshete (2006) who reported 6\% and 29.08\% seroprevalence respectively. Moreover, Gelagay Ayelet et al. (2007) have also reported prevalence of $20.12 \%$ from selected districts of Borana pastoral areas. Sharew et al. (2005) have also reported a seroprevalence of $52 \%-100 \%$ in acute outbreak areas using serological tests such as CFT and B-ELISA. The variation in the seroprevalence could be attributed to study design sampling strategy, sample size, purpose of the study and difference in study area as compared to the present study.

It was observed that the prevalence of the diseased increases as animals gets older. The observation of the community as shown in the questionnaire survey also agrees with the result of the serological findings in such a way that the occurrence of the disease increases with increasing age. The finding of this study contradicts with the works of Daniel Gizawu et al. (2009), but agrees with the report of Gezahegn Eshete (2006) who observed the presence of significant variation among age groups. However, different authors have indicated that as the goats get older; they are more susceptible to diseases than young once (Mike, 1996). Moreover, from the questionnaire survey it was observed that as age increases, they are often frequently exposed to different stress conditions which make them more susceptible to infection. Moreover, they also tend to be infected repeatedly. Therefore, the probability to be seropositive in older ages for CCPP would be high as compared to young and adult goats. The difference in seroprevalence among the various flock sizes categories was significant $(p<0.05)$. The questionnaire survey also identified that large flock size was a factors in the spread and occurrence of the disease. This may be explained by the fact that the infection needs proximity to source of infection and increasing number of susceptible population. The aggregation of goat flocks during watering, grazing and rest times would favor the spread of the infection within the flock. In pastoral areas, the users of the static clinical service and veterinary extension system are mostly those communities residing within 
the distance of $10 \mathrm{kms}$ to $15 \mathrm{kms}$ from veterinary service centers. Therefore, most areas beyond this radius do not have access to the service. Therefore, the extension of veterinary service to such and inaccessible corners is very limited (MoARD, 2003). In this study the seroprevalence observed among the distance categories was significant and the prevalence of CCPP was higher in distant communities. Previous study by Teshale Sori (1999) in the area has indicated that an impact of insufficient veterinary service in mobile livestock production system increases the spread of diseases. This finding could be attributed to the inaccessibility of such areas to veterinary services targeted to the disease. In this study it was observed that age, flock size and distance from veterinary service centre were the major risk factors associated with the occurrence of CCPP. Therefore, these factors could be used to construct a model to predict the occurrence of the disease in the study area. In conclusion, the serological findings and questionnaire survey indicated that CCPP is the top major goat health problem in the area which warrants appropriate measures to be in place towards the prevention and control of the disease.

\section{Acknowledgements}

We thank the Faculty of Veterinary Medicine of Addis Ababa University, National Veterinary Institute, Oromia Pastoralist Areas Development Commission and Care Ethiopia for supporting the research work.

\section{References}

Ayelet, G., Sori, T., Amsalu, W. and Gelaye, E., 2007. Prevalence of contagious caprine pleuropneumonia in the Borana pastoral areas of Ethiopia. Small. Rumin. Res., 70: 131-135.

Coppock, D. L., 1994. The Borana Plateau of Southern Ethiopia: Synthesis of Pastoral Research, Development and Change. Synthesis Study No 5. International Livestock Centre for Africa (ILCA), Addis Ababa, Ethiopia, pp. 1980-1991.

Eshete, G., 2006. Serological and Participatory Epidemiological Survey of Contagious Caprine Pleuropneumonia in Afar Pastoral areas of North East Ethiopia. In: Abstracts of MSc Theses (1998-2006). Faculty of Veterinary Medicine, Addis Ababa University, Debre Zeit.

Gizawu, D., Gebre-Egziabeher, B., Ayelet, G. and Asmare, K., 2009. Investigation of Mycoplasma infection in goats slaughtered at ELFORA export abattoir, Ethiopia. Ethiop. Vet. J.13 (1), 41-58.

Ethiop. Vet. J., 2011, 15 (2), 69-76 
Mekuria, S., and Asmare, K.., 2009. Cross sectional study on Contagious Caprine Pleuropneumonia in selected districts of Sedentary and Pastoral Production systems of Southern Ethiopia. Ethiopia. Ethiop. Vet. J.13 (2), 35-48.

Mike, S., 1996. Goats: In the Tropical Agriculturalist. Macmillan. 1st Edition, Malaysia, pp. $79-83$.

Ministry of Agriculture and Rural Development (MoARD), 2003. Pastoral Areas Development Study (PADS). In: Animal Health Thematic Report, Number 4, Rome and Addis Ababa, pp. 1-114.

Nicholas, R. A. J., 2002. Contagious Caprine pleuropneumonia. In: Tempesta M. (Ed.), Recent Advances in Goat Disease. International Veterinary Information Service (IVIS), Ithaca Ny (www.ivis.org.); A0907-0802: available at file: //A: \CCPP.HTM accessed on 20/04/2008 pp. 1-6.

Nigatu, B., 2003. Epidemiology and Serosurveillance of Contagious Caprine Pleuropneumonia in Dire Dawa Administrative Region. Vet. Rec., 131, 585-600.

OIE, 2004. Contagious Caprine Pleuropneumonia. Manual of Standards for Diagnostic Tests and Vaccine for Terrestrial Animals. Office International Des Epizooties (OIE). 5rd ed. V. II, Paris, France. pp. 623-632.

Radostitis, O. M., Gay, C. C, Hinchliff, W.K. and Constable, D. P., 2006. Veterinary Medicine. A Text Book of the Disease of Cattle, Sheep, Pigs, Goats and Horses. 10th Edition, W.B. Saunders Company Ltd. London: pp. 1140-1141.

Sharew, A. D., Staak, C., Thiacourt, F., and Roger, F., 2005. A Serological investigation into contagious caprine pleuropneumonia (CCPP) in Ethiopia. Trop. Anim. Hlth. Prod., 37, 11-19.

Sori, T., 1999. Ethno Veterinary Practice of the Borana Rangeland Pastoral System. In: Abstracts of DVM Theses (1985-2007). Faculty of Veterinary Medicine, Addis Ababa University.

SPSS, 2006. Statistical Package for Social Sciences. Version 15.0, SPSS Inc, 1989-2006, USA.

Thiaucourt, F., Bread, A., Lefevre, P. C., Meberatu, G. Y., 1992. Contagious Caprine pleuropneumonia in Ethiopia. Vet. Rec., 131 (25/26): 585; 3 ref. 